\title{
El liderazgo y la gestión en la solución de problemas perversos. Una revisión de la literatura
}

\author{
Jaime A. Riquelme-Castañeda ${ }^{\text {* }}$, Liliana M. Pedraja-Rejas ${ }^{2}$, y Roberto A. Vega-Massó1 \\ (1) Centro de Liderazgo, Universidad Finis Terrae, Pedro de Valdivia 1509, Providencia- Chile \\ (e-mail: jriquelme@uft.cl: rvega@uft.cl) \\ (2) Ingeniería Industrial, Universidad de Tarapacá, 18 de septiembre 2222, Arica- Chile \\ (e-mail: Ipedraja@uta.cl)
}

* Autor a quien debe ser dirigida la correspondencia

Recibido Feb. 25, 2019; Aceptado Abr. 21, 2019; Versión final Jun. 30, 2019, Publicado Feb. 2020

\begin{abstract}
Resumen
En este trabajo se analiza, desde una perspectiva conceptual, el liderazgo y la gestión, desde la cultura organizacional y la toma de decisiones. Esto tiene como objetivo estructurar un modelo integrador frente a la solución de problemas perversos. Se recurre a fuentes secundarias cuyo análisis e integración favorece una propuesta teórica preliminar que relaciona diferentes dimensiones. Como resultado, se propone un modelo de liderazgo integrador que sugiere relaciones sistémicas entre la composición del equipo directivo, la cultura organizacional, la relación con el escalafón superior, el proceso de toma de decisiones y los estilos de liderazgo transformacional y transaccional. Se evidencia un probable vínculo entre la efectividad del liderazgo y las condiciones sistémicas, encontrando, a partir de la metodología empleada, una asociación de naturaleza teórica no observada.
\end{abstract}

Palabras clave: liderazgo; organizaciones; gestión; cultura; toma de decisiones

\section{Leadership and management in wicked problem solving. A literature review}

\begin{abstract}
This paper analyzes the literature on leadership and management, from the organizational culture and decision making. This is done to develop an integrating model of organizational leadership to solve wicked problems. Secondary sources are reviewed and analyzed to make a preliminary theoretical proposal that integrates different dimensions. As a result, an integrating leadership model that suggests a systemic relationship between the managerial team composition, organizational culture, relationship with superiors, decision making process and the transformational and transactional leadership styles is proposed. The results show a probable link between leadership effectiveness and the systemic conditions finding, through the methodology used, a relation of theoretical nature, not observed before.
\end{abstract}




\section{INTRODUCCIÓN}

La globalización y la revolución tecnológica han provocado que el entorno de las organizaciones sea cada vez más complejo y que esta complejidad sea a la vez más robusta (Hitt, 1998). En la actualidad, pareciera ser más necesario aceptar que esta genera niveles crecientes de incertidumbre y amenazas producto de las paradojas y las contradicciones que se develan al tratar de comprender los problemas, describir sus variables, explicar su comportamiento y predecir su futuro (Fraher y Grinth, 2018; Fraher et al., 2017). Cuando se logra comprender una parte del problema, casi sin aviso las variables mutan y el problema cambia dejando tras de sí aquellas interpretaciones y soluciones que dan certeza. Este tipo de problemas, denominados perversos, son cada vez más comunes y se caracterizan por su complejidad, a veces inabordable, por demandar soluciones no lineales, que generan otros problemas, y por provocar gran incertidumbre (Grinth, 2005). Estos se consideran difíciles de comprender porque no se logra visualizar claramente sus componentes, establecer sus relaciones, ni predecir su comportamiento y las decisiones que se toman, para solucionarlos, generan problemas subsecuentes en las organizaciones. La eficiencia frente a estos problemas es casi inalcanzable y los decisores más experimentados aprenden a conformarse, al menos inicialmente, con la eficacia.

La proliferación de problemas perversos está demandando, en las organizaciones, el cuestionamiento y la adaptabilidad permanente de la estructura y de la cultura, convirtiéndolas en entidades hipercomplejas cada vez más difíciles de manejar (Ganga et al., 2017). Con tal de asegurar la supervivencia, las organizaciones han pasado de ser un ente ordenado que produce certezas, a quienes la integran, a un ente caótico que genera incertidumbres. La gestión, como representación de una alta evitación de incertidumbre, está dando actualmente respuestas adecuadas sólo en contextos de burocracia y de crisis (Osborn et al., 2002). El liderazgo, por otro lado, como representación de una baja evitación de incertidumbre, se ha convertido en un factor que, cada vez más, favorece el éxito de las organizaciones en contextos de complejidad (RodríguezPonce y Pedraja-Rejas, 2017; Uhl-Bien et al., 2007; y Grinth, 2005). La gestión y el liderazgo, en la evitación y aceptación de la incertidumbre, representan el dilema de la conservación y de la transformación, respectivamente, y saber cuándo optar más por una que por la otra, la decisión más difícil.

La toma de decisiones es un proceso clave para abordar la solución de los problemas perversos. En este proceso, la evitación de incertidumbre resulta comprensible, pero este tipo de problemas no ofrece muchas veces la cantidad suficiente de datos para obtener certezas. Está siendo necesario, cada vez más, atreverse a tomar decisiones con aceptación de la incertidumbre y, por tanto, los modelos normativos de optimalidad están dando paso a los cada vez más valorados modelos descriptivos de satisficiencia (Altman, 2008). La eficacia (búsqueda de la satisficiencia) y la eficiencia (búsqueda de la optimalidad) se están convirtiendo en un efecto de la toma de decisiones que, según el contexto, puede favorecer secuencialmente la solución final de un problema perverso. Contentarse inicialmente con una solución viable (eficacia) y más tarde con una óptima (eficiencia) se hace necesario para abordar la transformación organizacional y la consecuente supervivencia frente a problemas perversos.

Aceptar la eficacia inicial y esperar activamente la tan anhelada eficiencia requiere arriesgarse en el presente, aceptando la incertidumbre en el futuro. Esta necesidad propia de la toma de decisiones puede explicarse desde la cultura organizacional. La evitación de incertidumbre que, junto a otras dimensiones culturales relacionadas con el liderazgo como la distancia de poder y el colectivismo, es una característica cultural que distingue a las sociedades latinoamericanas y que explica, de alguna manera, no solo el comportamiento de los grupos humanos al interior de las organizaciones, sino además los bajos índices de innovación, de desarrollo científico y tecnológico y de respeto por la propiedad intelectual en esta región. La sociedad chilena tiene un $86 \%$ de evitación de incertidumbre lo que es alto si se compara con el $58 \%$ que promedian los diez países con mejores índices de competitividad, desarrollo humano y equidad de la Organización para la Cooperación y el Desarrollo Económicos- OCDE (Hofstede et al., 2015). Estos altos niveles de evitación de incertidumbre explican la conservación de las estructuras, la mantención dentro de lo conocido y la predilección por aquellas definiciones claras de las acciones y procedimientos en las organizaciones chilenas (Didier y Luna, 2017). El líder desempeña un rol esencial en las organizaciones ya que es quien influye para que los miembros de dicho equipo o de la organización lleven a cabo las tareas requeridas para conseguir los objetivos propuestos (Bernasconi y Rodríguez-Ponce, 2018).

En este contexto, el liderazgo es una herramienta que favorece la solución de los problemas perversos (Fraher y Grinth, 2018; Grinth, 2005). Los estilos de liderazgo, como patrones de conducta, tienen una incidencia fundamental sobre la eficiencia y la eficacia en los procesos estratégicos (Rodríguez- Ponce, 2007; PedrajaRejas y Rodríguez- Ponce, 2008; y Pedraja- Rejas et al., 2008). Existe un estilo de liderazgo que por un lado resulta apropiado para la solución de problemas futuros, normalmente difíciles de percibir, y otro estilo, que favorece la solución de problemas presentes, que son más evidentes. El primero favorece la transformación y el cuestionamiento del estatus quo y el segundo, la transacción y la conservación de éste (Bass, 1997). 
El dilema de qué transformar y qué conservar en las organizaciones se está convirtiendo tal vez en su principal desafío y las habilidades de liderazgo para diseñar e implementar la estrategia más efectiva, en una importante brecha de formación humana. Las habilidades de liderazgo o también conocidas como habilidades sociales, son las más valoradas en las organizaciones en Latinoamérica y especialmente en Chile en comparación con las habilidades técnicas y habilidades de conceptualización (Bassi et al., 2012). En los países de la región se valora más el liderazgo que los conocimientos y las técnicas, pero la educación escolar y el esfuerzo de formación que se realiza, especialmente en el caso de Chile, no privilegia el desarrollo de éstas sino la adquisición de conocimientos y aplicación de técnicas (Reimers y Chung, 2016). La formación escolar y universitaria, así como la capacitación de ejecutivos requieren dar pasos decididos hacia la formación de habilidades de liderazgo, toda vez que se necesita desarrollar habilidades que favorezcan la solución de los cada vez más frecuentes problemas perversos.

Los problemas perversos están desafiando la dirección de las organizaciones que luchan por mantener su supervivencia. La evitación de incertidumbre es determinante en el dilema del liderazgo y la gestión. El liderazgo, que acepta la incertidumbre, favorece la transformación y la eficacia. La gestión, que evita la incertidumbre, favorece la conservación y la eficiencia. Una adecuada combinación de liderazgo y gestión, que, desde un enfoque sistémico, no agota la diversidad de componentes del problema que en sí implica la toma de decisiones, permite lograr la eficacia y la eficiencia y por tanto, asegura la supervivencia de las organizaciones.

Los antecedentes presentados, dejan en evidencia la pertinencia y la importancia del liderazgo para explicar la eficacia de las organizaciones en contextos de mayor complejidad e incertidumbre (Rodríguez- Ponce, 2007). Se hace necesario entonces comprender el liderazgo como una herramienta que favorece la solución de los problemas perversos que actualmente afectan con mayor frecuencia a las organizaciones y con dicho propósito este trabajo presenta una perspectiva sistémica de aquellos aspectos de la teoría del liderazgo que mejor explican su relación con la eficacia de las organizaciones frente a los problemas perversos. Avanzar en esta comprensión contribuirá a que las organizaciones aseguren su supervivencia en tiempos de turbulencia y su efectividad en momentos de calma.

\section{OTROS ANTECEDENTES}

El liderazgo es el arte de solucionar problemas relevantes con una actuación armonizada de grupos humanos. Estos problemas, perversos por excelencia, son el tipo de escenario que favorece la comprensión del liderazgo, e indagar sobre estos es una labor necesaria y altamente atractiva (Ganga y Navarrete- Andrade, 2014). Son múltiples las indagaciones que han dejado de manifiesto la existencia de una gran cantidad de definiciones de liderazgo (Botero, 2018). Esta cantidad de información pareciera confundir más que aclarar el concepto.

El modelo interaccional se estima como una propuesta que facilita la comprensión del liderazgo (Hollander, 1978). El liderazgo es un proceso que resulta de una interacción armónica entre un líder, sus colaboradores y una situación que es necesaria de liderar, por ejemplo, un problema perverso. Este modelo da cuenta de la importancia que tienen las relaciones y las decisiones en un proceso de liderazgo. En ese sentido, las relaciones y las decisiones que surgen de las interacciones entre el líder, los colaboradores y la situación son claves para la efectividad del liderazgo. Para comprender integralmente la relación entre el líder, los colaboradores y la situación, se explican los estilos de liderazgo, la composición del equipo directivo, la cultura organizacional, la relación con el escalafón superior, así como el proceso de toma de decisiones.

\section{Los estilos de liderazgo}

De las diversas teorías asociadas a los estilos de liderazgo, la que ha llegado a convertirse en la más citada resulta ser la teoría de liderazgo transformacional y transaccional de Bass y Avolio (Pedraja- Rejas et al., 2018). Bajo esta teoría, los estilos de liderazgo transformacional y transaccional favorecen la transformación y la conservación del estatus quo, respectivamente. El primero reúne las características idealizadas de lo que tradicionalmente las personas entienden por liderazgo y el segundo, más bien las características de lo que se entiende por gestión (Bass, 1997). Estos estilos de liderazgo relacionan al líder con los colaboradores y con la situación desde la motivación y la efectividad en un contexto de toma de decisiones. Si bien inicialmente se sostuvo que ambos estilos son mutuamente excluyentes, hoy se demuestra que son complementarios y que el liderazgo transformacional es ineficaz sino tiene una base de liderazgo transaccional y de esta manera un líder que presenta características de ambos estilos podría alcanzar mayores niveles de desempeño (Laohavichien et al., 2009).

El liderazgo transformacional se define como la operación que se diseña e implementa para solucionar problemas futuros o difíciles de percibir, incentiva la adaptabilidad y busca nuevas formas de trabajo (Bass, 
1997). Agrega que asume los riesgos, energiza al equipo para persistir cuando las condiciones son impredecibles, difíciles y estresantes. Tiene menos apego al estatus quo. Es propio de una cultura organizacional colaborativa en la que se comparten objetivos comunes.

El liderazgo transaccional se define como la operación que se enmarca en el sistema o cultura organizacional existente, que evita el riesgo, es prisionera de los límites de tiempo, es altamente regulada, y a través del seguimiento y el control de los colaboradores está en permanente búsqueda de la eficiencia (Bass, 1997). Sus métodos de toma de decisiones más usados son normalmente racionales o económicos. Es propio de una cultura organizacional competitiva en la que se lucha por el posicionamiento al interior de una organización y se evidencia cuando los seguidores están de acuerdo, aceptan y cumplen con el líder a cambio de elogios, recompensas, entrega de recursos o la evitación de la acción disciplinaria (Bass et al., 2003).

Los estilos de liderazgo mejoran la flexibilidad del proceso de toma de decisiones mediante una mayor apertura de ideas, una mayor discusión y la posibilidad de ser creativos (Sharfman y Dean, 1997). El estilo de liderazgo transformacional se correlaciona positiva y significativamente con la flexibilidad. Ésta a su vez, se correlaciona positiva y significativamente con la racionalidad del proceso de toma de decisiones (RodríguezPonce, 2007). Entonces, el liderazgo transformacional mejora la racionalidad de las decisiones mediante una adecuada flexibilidad en el proceso de toma de decisiones.

La proposición más importante de la teoría transformacional y transaccional en la actualidad es el efecto de aumento, el cual estipula que el estilo de liderazgo transformacional se suma al efecto del estilo de liderazgo transaccional y que juntos producen un liderazgo efectivo (Anderson y Sun, 2017). Esto se describe como el grado en que el liderazgo transformacional se apoya sobre el liderazgo transaccional para contribuir a la eficacia de la organización (Bass, 1998). Es por esa razón que se considera que ambos estilos son complementarios. Incluso se sostiene que el liderazgo transformacional no es posible de ejercer sin el liderazgo transaccional, ya que la aplicación de este último por parte del líder produce una base de confianza, fiabilidad y percepción de coherencia en los seguidores.

En ambientes de gran incertidumbre, que requieren la ejecución de muchos procedimientos complejos, se aumenta la importancia requerida del liderazgo transaccional porque da estructura y estándar al desempeño de organizaciones de corta duración, asegurándose la efectividad de la operación, en comparación con ambientes menos complejos. Los hallazgos en esta materia son transferibles a cualquier tipo de organización que se desenvuelva en ambientes igualmente dinámicos (Bass et al., 2003).

Los estudios demuestran que, para liderar en situaciones de incertidumbre y estrés, como un proceso de transformación o de innovación, si bien se necesita una mayor relevancia del liderazgo transformacional se necesita también una sólida base de liderazgo transaccional que establezca estándares, expectativas de desempeño claras y sirva de base para la confianza en el líder (Ahmad et al., 2018; Tepper et al., 2018; Bass et al., 2003). El liderazgo transformacional puede basarse en estos niveles iniciales de confianza y aumentar la potencia y cohesión mediante un fortalecimiento de los valores, la misión de la organización y la visión (Shamir et al., 1998). El liderazgo transformacional, a pesar de responder a un prototipo idealizado o teoría implícita en diferentes culturas, no sustituye al liderazgo transaccional, sino que lo mejora (Bass, 1997). Incluso en situaciones de alta incertidumbre y estrés ambos estilos son igualmente significativos para asegurar la efectividad de una organización en una operación de corta duración y que el liderazgo transformacional pasa a ser más significativo en la medida que esta operación se hace más duradera (Bass et al., 2003).

\section{La composición del equipo directivo}

El liderazgo se puede explicar, por las características del equipo directivo (líder y colaboradores), desde la identificación de un problema hasta la toma de una decisión. Cuando los directivos integran su comportamiento, es decir, trabajan en equipo, involucrándose en una interacción mutua y colectiva (comparten información, recursos y decisiones) logran un mejor desempeño, adquiriendo especial relevancia la composición del equipo (Hambrick, 2007). La composición del equipo directivo, por ejemplo, el desarrollo de la carrera profesional y los niveles de educación, son relevantes para los resultados organizacionales porque la base cognitiva y valórica de éste es una pantalla entre la situación real y su eventual percepción (Hambrick, 2007).

Los equipos directivos de alta heterogeneidad educativa logran decisiones de mayor calidad en un contexto de complejidad e incertidumbre, que no alcance a constituir una crisis, porque logran una mayor sensibilidad para enfrentar la complejidad del entorno, están más condicionados para dar sentido a las situaciones cambiantes, conciliando los conflictos y las paradojas y tienen una mayor capacidad para superar la sobrecarga de información (Carpenter y Fredrickson, 2001). La diversidad en equipos directivos demuestra estar relacionada con un mejor desempeño organizacional ante los desafíos de la sustentabilidad ya que 
promueve una gestión multidimensional (Henry et al., 2019). La interdisciplinariedad de estos equipos favorece una mayor diversidad de opiniones, conocimientos e ideas, porque existe una más amplia diferenciación de experiencias, habilidades y redes de información, generándose más creatividad y agilidad en la resolución de problemas (Athanassiou y Nigh, 1999). El liderazgo transformacional por su parte se estima como un factor moderador que mejora el desempeño de equipos directivos diversos, fortaleciendo la identidad y la reflexión colectiva, flexibilizando la obtención de información y promoviendo interacciones positivas entre los miembros del equipo (Guillaume et al., 2017).

\section{La cultura organizacional}

El conjunto de prácticas, valores y creencias compartidas en un equipo directivo constituye la cultura organizacional. Esta cultura, y la conducta asociada, es más relevante para la toma de decisiones y los resultados organizacionales que una búsqueda mecánica de optimización económica, especialmente en situaciones de complejidad e incertidumbre (Hambrick, 2007). La cultura organizacional se construye paulatinamente a través de las experiencias sociales que son la resultante de múltiples intentos tanto de éxito como de fracaso. De este modo, mediante un aprendizaje social, que se transmite de generación en generación, se va conformando las creencias, los valores y el conocimiento. Posteriormente, estas ideas o fundamentos son institucionalizados fijando un conjunto de leyes o normas que garantizan un comportamiento socialmente adecuado o correcto (Pedraja- Rejas et al., 2018).

La cultura puede ser medida mediante indicadores como la evitación de incertidumbre (Hofstede et al., 2010). Este indicador se entiende como el grado de amenaza percibida e incomodidad producida en las personas cuando se enfrentan a situaciones ambiguas, desconocidas o desestructuradas (Hofstede, 2001). En lo práctico representa cuánto se esfuerza una organización en controlar lo incontrolable (Hofstede, 2001). Las culturas organizacionales con alta evitación de incertidumbre, en oposición a las de baja evitación, se caracterizan por un mayor apego al estatus quo, y con ello, a la estructura, a las normas y al cumplimiento de procedimientos, precisamente para reducir dicha incertidumbre (Hofstede, 2001). El proceso de planificación busca conocer con precisión qué va a ocurrir antes de tomar una decisión, siendo altamente valorada la predicción de eventos futuros (Triandis, 2004). Es común que se demanden líderes más directivos y detallistas en la definición de roles y tareas, buscando disminuir la ansiedad propia y de los colaboradores; y especialmente en los escalafones inferiores, la ansiedad es mitigada mediante el paternalismo que actúa como refugio para evitar la incertidumbre (Rodríguez y Ríos, 2007). La cultura con alta evitación de incertidumbre tiende a inhibir la libertad de acción de los equipos directivos, impidiendo un mayor aprovechamiento de las oportunidades en el entorno (Hambrick, 2007; Hofstede, 2001).

\section{La relación con el escalafón superior}

La presión por el desempeño que ejerce el escalafón superior y la discrecionalidad otorgada al equipo directivo para decidir explican hasta un $20 \%$ de la calidad de la decisión y de los resultados organizacionales (Hambrick, 2007). A mayor libertad de acción y menor presión en el desempeño, aumenta la objetividad del equipo directivo y por tanto mejoran sus resultados (Hambrick, 2007; Cannella, 2001). Los valores y actitudes de los miembros del equipo directivo responden a las acciones que son recompensadas o castigadas por el escalafón superior. Por esta razón, la cultura suele ser una barrera para la innovación, aunque a través de un esfuerzo consciente y coordinado en el tiempo puede ser moldeada (Jensen y Meckling, 1994).

En este contexto, y considerando que las necesidades son ilimitadas y no siempre materiales, los individuos evalúan constantemente la situación y en consecuencia están dispuestos a sacrificar un poco de casi todo, incluso la reputación y la moral, por una cantidad de lo deseado, que no necesariamente es dinero o bienes materiales, sino, sobre todo, respeto, poder, amor y bienestar de los demás. En esta búsqueda permanente de la maximización, los individuos actúan con gran creatividad generando oportunidades donde no existen y queriendo aflojar las restricciones impuestas por la organización. Esta divergencia entre el equipo directivo (agente) y el escalafón superior (principal) se reconoce como problema de agencia (Jensen y Meckling, 1994).

\section{El proceso de toma de decisiones}

El comportamiento del equipo directivo, durante el proceso de toma de decisiones, es más determinante en la calidad de las decisiones cuando la situación es compleja y de gran incertidumbre. Los decisores no pueden alcanzar un dominio cognitivo y técnico óptimo para lograr una decisión correcta, ya que la comprensión de la situación que se alcanza acostumbra a ser incompleta, filtrada y altamente estilizada (Cannella, 2001).

La base cognitiva y valórica, o idiosincrasia del equipo directivo, filtra y distorsiona la percepción de la realidad, limitando el campo de visión, seleccionando la información que se percibe y luego interpretando lo que está pasando y lo que se debe hacer al respecto (Hambrick y Snow, 1977). La base cognitiva es el conjunto de 
suposiciones que se tienen respecto del desarrollo de eventos futuros, de las alternativas de solución al problema planteado y de las consecuencias de alternativas de solución definidas. La base valórica es el conjunto de priorizaciones que se tienen en cuanto a la importancia de las alternativas de solución y sus consecuencias. En este sentido, la Teoría de Escalafones Superiores propone que los resultados de la organización, generados por las elecciones estratégicas y el desempeño, están predeterminados de forma concreta por el equipo directivo (Hiebl, 2014; Waldman y Javidan, 2013).

Ahora bien, en un contexto de complejidad e incertidumbre, como es característico de un problema perverso, los decisores se esfuerzan en la búsqueda de soluciones satisficientes o viables más que óptimas, decidiendo por la primera alternativa que dé solución al problema, porque se comportan como seres satisficientes (satis de satisfactorio y ficiente de suficiente) más que optimizadores, al reconocer la necesidad de luchar por la supervivencia más que por la maximización. Esta satisficiencia y optimalidad pueden ser igualmente efectivas si se comprende adecuadamente el entorno y se opera con ambas de forma unificada (Kruglanski y Gigerenzer, 2011).

En la búsqueda de soluciones viables y de decisiones oportunas y correctas adquiere especial relevancia la representación, es decir, la idea o imagen que se tiene de la realidad. Esta representación se logra a través del aprendizaje organizacional y éste, del aprendizaje individual, adquiriendo gran relevancia el intercambio de conocimiento, el respeto por la experiencia y el reconocimiento de la voluntad de cada decisor que integra el equipo directivo (Simon, 1991). Los sesgos cognitivos, que dan base a esta representación y que pueden distorsionar la toma de decisiones, son más fáciles de ser detectados en otra persona que en sí mismo, razón por la que adquiere especial relevancia la toma de decisiones en equipos directivos por sobre la toma de decisiones de un directivo aislado, en situaciones de mayor incertidumbre (Barón y Zapata, 2018). Los sesgos cognitivos se valoran más como una ventana al procesamiento de información racional que un signo de irracionalidad humana (Lieder et al., 2018).

Es importante estimar el costo de deliberación para decidir si se aplican métodos de racionalidad limitada (satisficiencia) o de racionalidad clásica (optimalidad). El asumir o no los costos de deliberación depende de la valoración de cada decisor, de la evitación de incertidumbre, de la obediencia o docilidad a las normas sociales y de la etapa y ámbito del proceso de toma de decisiones, aunque se estima que a mayor costo de deliberación la racionalidad limitada adquiere mayor relevancia (Kahneman, 2003).

La racionalidad limitada en ambientes de complejidad y de alta incertidumbre, así como en procesos de aprendizaje y adaptación, produce decisiones más exitosas que la racionalidad clásica si se fortalece la experiencia previa, se practica en forma permanente y se asumen altos riesgos. Sin embargo, en la actualidad se ha transitado hacia un único sistema cognitivo de plena complementación, sosteniéndose que una racionalidad sensata, entendida como una aplicación adecuada de ambas metodologías según costos de deliberación, complejidad, incentivos, experiencia y normas sociales, favorece el éxito de las decisiones (Kahneman, 2003).

Finalmente, resulta necesario indicar que, bajo esta lógica de análisis, se evidencia que los estilos de liderazgo transformacional y transaccional son mutuamente necesarios y, dependiendo de la situación, uno adquiere mayor relevancia que el otro e incluso en situaciones de estrés e incertidumbre ambos pueden ser igualmente importantes. El estilo de liderazgo depende de la situación que el líder tiene que resolver, no siendo lo mismo solucionar un problema bien estructurado que resolver problemas difusos y mal definidos como una crisis (Antonakis et al., 2003). Por tanto, en la medida que los decisores desarrollan la capacidad de aplicar ambos estilos de liderazgo de manera adecuada, el liderazgo en el equipo directivo se relaciona con la efectividad organizacional en situaciones de complejidad e incertidumbre, como un problema perverso.

A partir del análisis conceptual realizado, en la figura 1 se muestra, desde una perspectiva sistémica, cómo sería posible asegurar la supervivencia de una organización frente a los problemas perversos. El entorno organizacional ofrece problemas perversos y exige supervivencia al mismo tiempo. En este contexto, el equipo directivo, encargado de solucionar dicho problema y de asegurar esta supervivencia, se relaciona con un escalafón superior al que rinde cuentas y esta relación puede favorecer su efectividad. La cultura representada en este modelo por la evitación de incertidumbre, entre otras dimensiones y componentes, se relaciona con el proceso de toma de decisiones y, en éste, con la necesidad de liderar y gestionar el problema perverso. Si el problema ofrece certezas sobre la base de experiencias previas, de información real y de datos adecuados, el equipo decide gestionar el problema y en aquellas variables controladas que han demostrado buenos resultados, conservar el proceso y optimizarlo para alcanzar altos niveles de eficiencia. Al mismo tiempo, si el problema ofrece incertidumbre, es decir ausencia de experiencias previas, información ambigua y datos insuficientes, el equipo decide liderar y en aquellas variables no controladas en las que no se prevén buenos resultados, transformar el proceso y aceptar niveles de satisficiencia y eficacia. En la medida que el equipo directivo frente a un problema perverso adopte el estilo de liderazgo adecuado, es decir, gestione y lidere la 
certeza y la incertidumbre, respectivamente, será posible transformar lo necesario y conservar lo esencial, alcanzando niveles de eficacia y de eficiencia que permitan asegurar, con sensatez, la supervivencia de una organización.

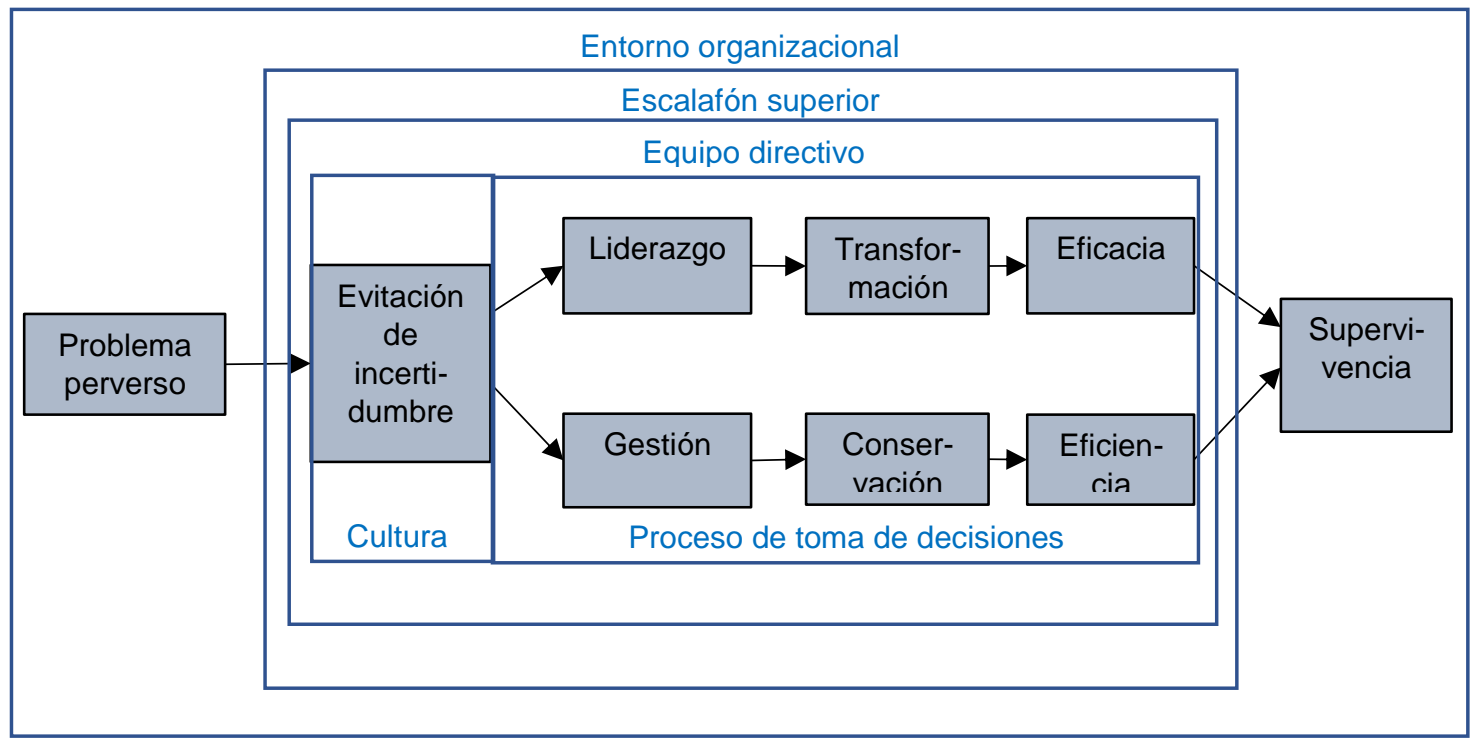

Fig. 1: El liderazgo y la gestión de problemas perversos

\section{DISCUSION FINAL}

El análisis conceptual llevado a cabo da cuenta que el liderazgo no depende sólo del líder ni exclusivamente de la relación de éste con sus colaboradores y la situación. Existen claras evidencias que el liderazgo es un proceso que forma parte de un sistema que condiciona su efectividad. El líder puede ejercer una influencia significativa, pero si el equipo directivo no tiene una composición adecuada en recursos y capacidades es probable que no logre un alto desempeño. En definitiva, la composición del equipo directivo es determinante para la efectividad del liderazgo. Si el equipo cuenta con colaboradores experimentados y con nóveles de alto nivel educacional, si además existe una amplia diversidad de disciplinas y buenas prácticas de trabajo en equipo, lograrán un mejor desempeño (Kane et al. 2018).

La relación con el escalafón superior es también determinante en el liderazgo de un equipo directivo. Si el equipo goza de una baja presión por el desempeño y una gran libertad de acción o discrecionalidad y si está de acuerdo con los mecanismos de recompensas y castigos establecidos por la organización, tendrá un mejor desempeño. Así también, el proceso de toma de decisiones es un factor significativo en el liderazgo de un equipo directivo (Heavin y Power, 2018). Si se entrena una metodología de toma de decisiones que valora la racionalidad limitada y asume riesgos, según costos de deliberación, y al mismo tiempo favorece la objetividad, inhibiendo sesgos y promoviendo el intercambio de conocimientos y el respeto por la experiencia entre los miembros del equipo, las decisiones serán de mejor calidad (Kahneman, 2003).

La composición del equipo directivo, su relación con el escalafón superior y el proceso de toma de decisiones son factores relevantes, sin embargo, la cultura organizacional y los estilos de liderazgo que promueve son decisivas (Bernasconi y Rodríguez-Ponce, 2018). La evitación de incertidumbre es probablemente el factor que promueve o inhibe el liderazgo en un equipo directivo. Si la evitación de incertidumbre es alta, el equipo directivo podría preferir no ejercer liderazgo y promover una gestión de excelencia tendiente sólo a resultados inmediatos y evidenciables (Hofstede, 2011). Si la evitación de incertidumbre es baja es probable que el equipo directivo se arriesgue a ejercer un liderazgo sensato que transforma lo necesario y conserva lo esencial, demostrando un justo equilibrio entre la conservación con eficiencia y la transformación con eficacia.

Se avanza en comprender que el liderazgo es una herramienta que favorece la solución de problemas perversos, caracterizados por altos niveles de complejidad e incertidumbre, y asegura la supervivencia de la organización, pero que éste es un proceso que depende de un sistema organizacional que condiciona su efectividad. Las organizaciones necesitan promover un sistema que favorezca una estructura y una cultura que acepta el riesgo de ejercer liderazgo y no se limita a una gestión de resultados mediáticos e inmediatistas (Fraher y Grinth, 2018). Finalmente, para fortalecer el liderazgo y, mediante éste, favorecer la solución de los problemas perversos se necesita una educación país que promueva, desde temprana edad, conductas que acepten la incertidumbre (Riquelme y Predraja, 2019). 


\section{CONCLUSIONES}

De acuerdo con el trabajo presentado y los resultados obtenidos, se pueden plantear las siguientes conclusiones principales:

i) La adecuada combinación de estilos de liderazgo favorece la solución de problemas perversos y la supervivencia de las organizaciones.

ii) El liderazgo y la gestión, como aplicaciones de los estilos de liderazgo transformacional y transaccional respectivamente, favorecen una sensata transformación y conservación de las organizaciones y una pertinente eficacia y eficiencia de los procesos.

iii) La efectividad del liderazgo en la solución de problemas perversos se vincula probablemente a condiciones sistémicas tales como la composición de los equipos directivos, la cultura organizacional, la relación con el escalafón superior, el proceso de toma de decisiones y los estilos de liderazgo transformacional y transaccional.

iv) Equipos directivos de alta diversidad que actúan con amplia libertad de acción, que aceptan y evitan la incertidumbre según la disponibilidad de información, que se disponen a transformar lo necesario y conservar lo esencial y que aplican métodos decisionales de satisficiencia y optimalidad favorecerían la supervivencia de las organizaciones en situaciones de alta complejidad.

\section{AGRADECIMIENTOS}

Los autores agradecen el apoyo de la Universidad Finis Terrae, a través del Centro de Liderazgo, y de la Universidad de Tarapacá, a través del proyecto mayor UTA 8740-16.

\section{REFERENCIAS}

Ahmad, N., C. Yanxia y otros tres autores, The influence of transactional leadership on innovative work behavior-a mediation model, European Journal of Business and Social Sciences, ISSN: 2235 -767X, 7(1), 51-62 (2018)

Altman, M., Behavioral Economics, Economic Theory and Public Policy, doi: 10.2139/ssrn.1152105, Social Science Research Network, EE.UU (2008)

Antonakis, J., B. Avolio y N. Sivasubramamiam, Context and leadership: an examination of the nine-factor full-range leadership theory using the Multifactor Leadership Questionnaire, doi: 10.1016/S1048-9843(03)00030-4, The Leadership Quarterly, 14(3), 261-295 (2003)

Anderson, M. y P. Sun, Reviewing Leadership Styles: Overlaps and the Need for a New 'Full-Range' Theory, doi: 10.1111/ijmr.12082, International Journal of Management Reviews, 19(1), 76-96 (2017)

Athanassiou, N. y D. Nigh, The impact of U.S. company internationalization on top management team advice networks: A tacit knowledge perspective, doi: 10.1002/(SICI)1097-0266(199901)20:1<83::AID-SMJ10>3.0.CO;2-Y, Strategic Management Journal, 20(1), 83-92 (1999)

Barón, L. y G. Zapata, Los sesgos cognitivos: de la psicología cognitiva a la perspectiva cognitiva de la organización y su relación con los procesos de toma de decisiones gerenciales, doi: 10.22206/CYS.2018.V43l1.PP31-48, Revista Ciencia y Sociedad, 43(1), 31-48 (2018)

Bass, B., Does the Transactional-Transformational Leadership Paradigm Transcend Organizational and National Boundaries?, American Psychologist, 2, 130-139 (1997)

Bass, B., Transformational leadership: Industry, military and educational impact, Lawrence Erlbaum Associates, New Jersey, EE.UU (1998)

Bass, B., B. Avolio, D. Jung y Y. Berson, Predicting Unit Performance by Assessing Transformational and Transactional Leadership, doi: 10.1037/0021-9010.88.2.207, Journal of Applied Psychology, 88(2), 207-218 (2003)

Bassi, M., M. Busso, S. Urzúa y J. Vargas, Desconectados: Habilidades, educación y empleo en América Latina, BID, New York, EE.UU (2012)

Bernasconi, A. y E. Rodríguez-Ponce, Análisis Exploratorio de las Percepciones sobre los Estilos de Liderazgo, el Clima Académico y la Calidad de la Formación de Pregrado, doi: 10.4067/S0718-50062018000300029, Formación universitaria, 11(3), 29-40 (2018)

Botero, M., Lo que llamamos liderazgo: Consideraciones críticas de lo que ocurre en diversas organizaciones, Perspectivas Psicológicas, volúmenes 3- 4, año IV, 134-144 (2018)

Cannella, A., Upper echelons: Donald Hambrick on executives and strategy, doi: 10.5465/ame.2001.5229499, Academy of Management Executive, 15 (3), 36-44 (2001) 
Carpenter, M. y J. Fredrickson, Top management teams, global strategic posture, and the moderating role of uncertainty, doi: 10.5465/3069368, Academy of Management of Journal, 44(3) 533-546 (2001)

Didier, N. y J. Luna, ¿Dónde Estamos? La Cultura Laboral Chilena desde Hofstede, doi: 10.15446/rcp.v26n2.60557, Revista Colombiana de Psicología, 26(2), 295-311 (2017)

Fraher, A., L. Branicki y K. Grinth, Mindfulness in action: discovering how U.S. Navy Seals build capacity for mindfulness in high-reliability organizations (HROS), doi: 10.5465/amd.2014.0146, Academy of Management Discoveries, 3(3), 239261 (2017)

Fraher, A. y K. Grinth, Agonistic Governance: The antinomies of decision-making in U.S. Navy SEALs, doi: 10.1177/1742715016656649, Leadership, 14(2), 220-239 (2018)

Ganga-Contreras, F., L. Pedraja-Rejas, J. Quiroz-Castillo y E. Rodríguez-Ponce, Isomorfismo Organizacional (IO): Breves aproximaciones teóricas y algunas aplicaciones a la educación superior, Espacios, ISSN: 0798-1015, 38(20), 31-43 (2017)

Ganga-Contreras, F. y E. Navarrete-Andrade, Aportaciones teóricas significativas sobre el liderazgo carismático y transformacional, Revista Venezolana de Gerencia, ISSN: 1315-9984, 19(67), 456-476 (2014)

Grinth, K., Problems, problems, problems: The social construction of leadership, doi: 10.1177/0018726705061314, Human Relations, 58(11) 1467-1494 (2005)

Guillaume, Y., J. Dawson y otros tres autores, Harnessing demographic differences in organizations: What moderates the effects of workplace diversity?, doi: 10.1002/job.2040, Journal of Organizational Behavior, J. Organiz. Behav, 38(2), 276$303(2017)$

Hambrick, D. y C. Snow, A contextual model of strategic decision making in organizations, doi: 10.5465/ambpp.1977.4977040, Academy of management Proceedings, 1, 109-112 (1977)

Hambrick, D., Upper Echelons Theory: An Update, doi: 10.5465/amr.2007.24345254, The Academy of Management Review, 32(2), 334-343 (2007)

Heavin, C. y D. Power, Challenges for digital transformation - towards a conceptual decision support guide for managers, doi: 10.1080/12460125.2018.1468697, Journal of Decision Systems, 27:sup1, 38-45 (2018)

Henry L. A., T. Buyl y R.J.G. Jansen, Leading corporate sustainability: The role of top management team composition for triple bottom line performance, doi: 10.1002/bse.2247, Business Strategy and the Environment, 173-184 (2019)

Hiebl, M., Upper Echelons Theory in Management Accounting and Control Research, doi: 10.1007/s00187-013-0183-1, Journal of Management Control, 24(3), 223-240 (2014)

Hitt, M. A., Presidential address: Twenty-first century organizations: Business firms, business schools, and the academy, doi: 10.5465/amr.1998.533223, The Academy of Management Review, 23(2), 218-224 (1998)

Hollander, E.P., Leadership Dynamics, 6ª Ed., Free Press, New York, EE.UU (1978)

Hofstede, G., Culture's Consequences: Comparing Values, Behaviors, Institutions and Organizations Across Nations, $2^{\mathrm{a}}$ Ed., Sage, Thousand Oaks, EE.UU (2001)

Hofstede, G., Dimensionalizing Cultures: The Hofstede Model in Context, doi: 10.9707/2307-0919.1014, Online Readings in Psychology and Culture, 2(1) (2011)

Hofstede, G., J. Hofstede y M. Minkov, Cultures and Organizations: Software of the Mind, 3ª Ed., McGraw-Hill, New York, EE.UU (2010)

Hofstede, G., J. Hofstede y M. Minkov, Culture and Organizations: Software of the Mind, Dimension Data Matrix, base de datos actualizada al 16-08-2015 (2015)

Jensen, M. y W. Meckling, The Nature of Man, doi: 10.1111/j.1745-6622.1994.tb00401.x, Journal of Applied Corporate Finance, 7(2), 4-19 (1994)

Kahneman, D., Maps of Bounded Rationality: Psychology for Behavioral Economics, doi: 10.1257/000282803322655392, The American Economic Review, 93(5), 1449-1475 (2003)

Kane, G., D. Palmer y otros tres autores, Coming of Age Digitally, MIT Sloan Management Review and Deloitte Insights, June (2018)

Kruglanski, A. y G. Gigerenzer, Intuitive and Deliberate Judgments Are Based on Common Principles, doi: 10.1037/a0023709, Psychological Review, 118(1), 97-109 (2011)

Laohavichien, T., L. Fredendall, \& R. Cantrell, The effects of transformational and transactional leadership on quality improvement. doi: 10.1080/10686967.2009.11918223, Quality Management Journal, 16(2), 7-24 (2009)

Lieder, F., T. Griffiths y M. Hsu, Overrepresentation of Extreme Events in Decision Making Reflects Rational Use of Cognitive Resources, doi: 10.1037/rev0000074, Psychological Review, 125(1), 1-32 (2018)

Osborn, R., J. G. Hunt y L. R. Jauch, Toward a contextual theory of leadership, doi: 10.1016/S1048-9843(02)00154-6, The Leadership Quarterly, 13(6), 797-837 (2002)

Pedraja-Rejas, L., J. Riquelme, R. Vega, La importancia de los estilos de liderazgo en la calidad de las unidades académicas universitarias, Opción, 34(86), 130-151 (2018) 
Pedraja-Rejas, L., C. Araneda-Guirriman, A. Bernasconi y P. Viancos, Liderazgo, cultura académica y calidad de las universidades: aproximación conceptual y relaciones, Revista Venezolana de Gerencia, ISSN: 1315-9984, Edición Especial 1, 184-199 (2018)

Pedraja- Rejas, L., E. Rodríguez-Ponce y J. Rodríguez-Ponce, Importancia de los estilos de liderazgo sobre la eficacia: un estudio comparativo entre grandes, medianas y pequeñas empresas privadas, Revista de Ciencias Sociales, ISSN: 1315-9518, 14(1), 20-29 (2008)

Pedraja- Rejas, L. y E. Rodríguez-Ponce, Estudio comparativo de la influencia del estilo de liderazgo y la congruencia de valores en la eficacia de empresas privadas e instituciones públicas, Revista Interciencia, ISSN: 0378-1844, 33(1), 8-13 (2008)

Reimers, F. y C. Chung, Enseñanza y aprendizaje en el siglo XXI, Fondo de Cultura Económica, 321 pp. México D.F. México (2016)

Riquelme-Castañeda J. y L. Pedraja-Rejas, El liderazgo, la cultura y los desafíos de la educación, doi: 10.4067/S071833052019000100005, Ingeniare, Revista Chilena de Ingeniería, 27(1), 5-7 (2019)

Rodríguez-Ponce, E. y L. Pedraja-Rejas, Relación entre el liderazgo transformacional y el clima orientado al servicio de los estudiantes: evidencia exploratoria desde Chile, Revista Interciencia, ISSN: 0378-1844, 42(10), 633-640 (2017)

Rodríguez-Ponce, E., Estilos de Liderazgo, Toma de Decisiones Estratégicas y Eficacia: Un Estudio Empírico en Pequeñas y Medianas Empresas, Revista Interciencia, ISSN: 0378-1844, 32(8), 522-538 (2007)

Rodríguez, D. y R. Ríos, Latent premises of labor contracts: paternalism and productivity, doi: 10.1108/01437720710778367, International Journal of Manpower, 28(5), 354-368 (2007)

Shamir, B., E. Zakay, E. Breinin y M. Popper, Correlates of charismatic leader behavior in military units: Subordinates' attitudes, unit characteristics, and superiors' appraisals of leader performance, doi: 10.5465/257080, Academy of Management Journal, 41(4), 384-409 (1998)

Sharfman, M. y J. Dean, Flexibility in strategic decision making: Informational and ideological perspectives, doi: 10.1111/1467-6486.00048, Journal of Management Studies, 34(2), 191-217 (1997)

Simon, H., Bounded Rationality and Organizational Learning, doi: 10.1287/orsc.2.1.125, Organization Science, 2(1), 125$134(1991)$

Tepper, B., N. Dimotakis y otros cinco autores, Examining follower responses to transformational leadership from a dynamic, person-environment fit perspective, doi: 10.5465/amj.2014.0163, Academy of Management Journal, 61(4), 1343$1368(2018)$

Triandis, H., The many dimensions of culture, doi: 10.5465/ame.2004.12689599, Academy of management executive, 18(1), 88-93 (2004)

Uhl-bien, M., R. Marion y M. McKelvey, Complexity Leadership Theory: Shifting leadership from the industrial age to the knowledge era, doi: 10.1016/j.leaqua.2007.04.002, The Leadership Quarterly, 18(4), 298-318 (2007)

Waldman D.A. y M. Javidan, Charismatic leadership at the strategic level: taking a new look at upper echelons theory; in: Transformational and Charismatic Leadership: The Road Ahead, 10ª Ed. by B.J. Avolio y F.J. Yammarino, pp 201-227, Emerald Group Publishing Limited, Bingley, UK (2013) 